\title{
El futuro de la psicoterapia: aprender de los errores ${ }^{1}$
}

RESUMEN: En la primera parte se descubren los errores teóricos-históricos de la investigación en psicoterapia familiar acerca de la "no existente" familia del esquizofrénico (o familia de la anoréxica). Esto es, el error consistente en buscar una correlación lineal entre síntoma y familia.

En la segunda parte se exponen los primeros datos de la evolución de los casos tratados por Mara Selvini durante los años setenta, siendo esos datos comparados con datos similares de anorexias.

PALABRAS CLAVE: personalidad, familia, trastorno antisocial, estudios de seguimiento, protocolos psicoterapéuticos.
SUMMARY: In the first part Matteo Selvini discusses the historical theoretical mistake of family therapy in investigating the "non existing" family of the schizophrenic (or family of the anorexic). It is the theoretical mistake of looking for a lineal link symptom and family.

The future is instead that of looking for a complex explanation of a less complex phenomenon loke personality traits.

In the second part Matteo Selvini proposes the first data ot the follow-up of psychotic cases treated by Mara Selvini Palazzoli during the seventies and these data are compared with similar data of anorexia follow-up.

KEY WORDS: personality, family, antisocial disorder, follow-up, psychotherapeutic protocols.

El ultimo libro de mi equipo con la participación activa de Mara Selvini Palazzoli, publicado en 1998 es Muchachas anoréxicas y bulímicas (1). En él hemos reflexionado sobre el límite teórico más importante en la historia de la psicoterapia familiar: la pretensión de identificar la familia de la anoréxica o la familia del psicótico.

\section{Crisis del modelo colectivo}

Así hemos escrito en el texto citado:

"Reflexionando a posteriori sobre algunas de nuestras experiencias insatisfactorias, nos vimos empujados a dar un salto cualitativo, llegando, al fin, a considerar ineludibles, a pesar del credo sistémico (pero no de una lógica relacional), las preguntas tradicionales sobre el "porqué" del pasado de los individuos, el funcionamiento defensivo y las

${ }^{1}$ Este trabajo corresponde a la ponencia presentada en las XVIII Jornadas de la AEN celebradas los días 5-7 de abril de 2002 en Huelva 
necesidades insatisfechas de cada uno (tanto de la paciente como de los padres), la relación entre tales necesidades y las experiencias de crianza y, en consecuencia, las relaciones de cada padre con su familia de origen. Este fue el momento, posterior a la prescripción invariable, de la integración, en nuestra aproximación, de las dimensiones individual y trigeneracional, desde una óptica nueva, que definimos como "pensar en idas y venidas" y pensamiento multidimensional complejo" (2: p. 96-97).

¿Qué entendemos por multidimensional? Trataremos de explicarlo.

La personalidad de cada sujeto, miembro de una familia, es apreciada en su individualidad en el aquí y ahora de su relación tanto con el terapeuta como con sus interlocutores familiares, además que con su expresión cotidiana. Este presente es fruto tanto de las relaciones en curso como de la historia de relaciones pasadas que han determinado la cristalización del actual equilibrio emotivo de la persona. (2:p-97)

En la investigación de los significados que hay que dar al síntoma y a las defensas de la paciente, es de una gran riqueza, conectar sus elecciones subjetivas con la historia familiar, todo ello en el entrelazamiento de las concomitancias temporales. Esta conexión debe ser concebida, empero, como rigurosamente específica de aquella paciente concreta y de aquella familia concreta: en efecto, nos parece que ahora estamos muy lejos de una óptica - que hemos abrazado largamente - demasiado "medicalista", que tendía a conectar una enfermedad con un agente patógeno: en este caso, un diagnóstico psiquiátrico, o bien una cierta constelación de síntomas, con un tipo reconocible de familia. Véanse, por ejemplo, los estudios de Lidz y sus colaboradores (3) sobre la esquizofrenia, los de Haley (4) sobre el mismo tema, o también los de Minuchin y sus colaboradores (5) y los de Selvini Palazzoli (6) sobre la anorexia, etc. (pp. 97-98).

La crisis de este modelo es la crisis de un modelo típicamente médico reduccionista: la investigación imposible de una causa simple y lineal para un fenómeno altamente complejo. Por ejemplo la familia esquizofrenógena que causa la esquizofrenia.

El futuro es en cambio el de encontrar explicaciones complejas para fenómenos relativamente simples de definir. Por ejemplo la falta de remordimiento que caracteriza a un delincuente es un fenómeno relativamente simple para el que tenemos buenas posibilidades de encontrar una explicación compleja, aunque convicente, demostrable y contestable.

Hoy ya no pensamos que el camino podría ser buscar rígidas tipologías de juegos familiares, como tampoco que exista "la personalidad de la anoréxica", a diferencia de ciertas reflexiones pasadas (6) y de lo sostenido por otros (véase, por ejemplo, Guidano y el concepto de "estructura TAP" (Trastorno Alimentario Psicógeno) (7). Del mismo modo, ya no pensamos que exista y deba buscarse "la familia de la anoréxica" (p. 98). 
Por tanto, nos parece evidente que la pretensión de identificar una "familia de la anoréxica" que se repita de un caso a otro es del todo imposible. Tratar de entender mejor es más complejo, aunque no debemos extraviarnos en esta complejidad, considerando que toda configuración es igualmente probable y, por tanto, todo descubrimiento es arbitrario. En cambio, en las familias de las anoréxicas, por ejemplo, se verifican dimensiones relacionales y familiares que son más recurrentes que otras: éstas son las redundancias personales, familiares y sociales que hemos creído verificar en nuestra casuística.

Hoy, pues, pensamos que podemos correlacionar más bien una cierta constelación de síntomas con una cierta personalidad de la paciente, por un lado, y con un cierto tipo de familia, por el otro.

Se debe trabajar sobre tres polos y no sobre dos. No puede haber una correlación directa entre el síntoma y la familia. ¿Por qué? Precisamente porque "en medio" está la compleja mediación de cómo un individuo, que dispone desde el nacimiento de sus propias características, elabora el sufrimiento y se defiende de el (en medio esta la relación de la persona con si misma).

Por eso, en la anorexia-bulimia encontramos familias muy distintas, que tienen hijas con personalidades igualmente distintas, que se sitúan en la vertiente dependiente, o borderline, u obsesivo- compulsiva, o narcisista. (pp. 98-99).

Volviendo, por tanto, a la triple polaridad sintomas-persona-familia, las conexiones básicas de nuestra investigación clínica serán entones:

a) Aquellas entre familias y personalidad individuales. En esto nos es de grandísima ayuda la investigación moderna que ha superado teorías desorientadoras del pasado (teoría de la pulsión de Freud, concepto de autismo primario ...), en favor de construcciones mucho más útiles para entender (las teorías de Bowlby sobre el apego, las de Kohut sobre la necesidades de empatía en el desarrollo del yo infantil, etc.). Es decir, en nuestra indagación comenzamos a rastrear de qué modo el individuo (al que suponemos en su nacimiento como único, singular e irrepetible), una vez que ha sido socialmente condicionado por las interacciones, elabora su experiencia primaria y, si ésta ha sido penosa, se defiende de ella.

b) En segundo lugar, las conexiones que buscaremos serán aquellas entre persona $y$ síntoma. Hoy estamos en condiciones de entender un poco mejor la conexión entre la persona del paciente, su modo de estar en el mundo, y la elección de un determinado síntoma, coherente con la estructura, a la vez, defensiva y expresiva de su "drama original".

La terapia familiar de la psicosis ha sido una terapia que ha trabajado sobre la idea de dar como primer mensaje a la familia que el síntoma es la expresión de un problema familiar o la tentativa de solucionar un problema familiar. La idea que hemos ensayado en tantísimas terapias familiar sistémicas es conectar el síntoma psicótico con 
un problema en la familia. Yo creo que empezar con esta idea en la terapia de la psicosis es peligroso en muchos casos, porque las familias de los psicóticos pueden no estar preparadas al principio del tratamiento para aceptar esta idea, al contrario se defienden de esta idea, un poco culpabilizadora. También implícitamente hay un primer paso que tenemos que hacer en el tratamiento de la psicosis y es el paso de ayudar a los padres a ver el sufrimiento del paciente. Las verdaderas razones personales del sufrimiento del paciente y cómo el síntoma representa una defensa para que el sufrimiento del paciente sea soportable y no conduzca a la muerte por suicidio u otra formas. Yo creo que antes debemos realizar esta etapa del tratamiento. El acuerdo de colaboración entre padres y hermanos sobre el sufrimiento del paciente, y después podemos (después puede ser un tiempo bastante largo) entrar en el tema relacional.

Raices relacionales del trastorno anti-social

Quisiera ahora simplificar estos conceptos teóricos hablando del caso de un joven delincuente. Michele, de 19 años, ha abandonado los estudios después de repetidos suspensos, no trabaja, sale con una banda a cometer robos y actos de vandalismo, roba sistemáticamente dinero a su madre y a los otros familiares.

El delito como síntoma es fácilmente asimilable a un trastorno de la personalidad antisocial, presenta de hecho casi todas las características descritas en el DSM IV.

Los padres, que están atravesando una pesada crisis como pareja (están recién separados) piden ayuda para el hijo, el cual rechaza cualquier consulta especializada. Mejor dicho, los padres hasta tienen miedo a decirle que están consultando con un experto. Temen su reacción de desprecio. El trabajo terapéutico permite encontrar toda una serie de conexiones muy claras entre la personalidad del chico y sus relaciones con los familiares.

Las esquematizo en áreas que creo generalizables en la casuística del trastorno antisocial de la personalidad:

1) Ninguno de los padres ha sabido ser para Michele una base segura, al contrario ambos le han exaltado dotes y capacidad tratándolo como un adulto mucho antes de tiempo. Michele ha desarrollado un apego evitativo. Un contexto relacional parecido se encuentra en el trastorno narcisista.

2) El padre, con graves carencias en el plano afectivo, se mantiene distante del hijo, percibido al menos en algunos momentos, como una especie de rival. Cuando la madre implica al padre para que castigue o riña al hijo Michele, el padre aprovecha para desencadenar la violencia, haciendo que la madre de la vuelta a la situación defendiendo al hijo (Cirillo, Rangone, Selvini, 9). Emblemático el episodio en el que mete la cabeza del hijo de 8 años en el water porque ha olvidado otra vez tirar de la cadena.

3) Ninguno de los padres, y en particular la madre, no son capaces de decirle no, es decir de contener al hijo poniéndole reglas y haciéndole respetarlas. La madre parece increíblemente cómplice de los robos que el hijo les hace sistemáticamente. Las relaciones familiares, de hecho, han sido siempre igualitarias - simétricas. 
4) Ambos padres tampoco han podido vivir, en sus respectivas familias, las relaciones empáticas, afectivas, cálidas, de colaboración. Las relaciones han sido siempre muy instrumentales y utilitaristas. Y esta dimensión parece ser también dominante en la familia de Michele.

5) Michele acaba teniendo una vivencia de sí mismo como víctima, tanto porque no consigue sentirse ligado a la madre y la vive como una estúpida, disponible a que le tomen el pelo, como porque siente al padre como distante y hostil. Esta vivencia de víctima viene elaborada en términos de autorización a resarcirse, obtener una compensación por el daño recibido: "tomaré yo solo aquello que no me viene dado". Esta vivencia de víctima justifica la característica clave del antisocial: la ausencia de remordimiento respecto a los actos delictivos. La vivencia de víctima viene alimentada por un instigador (en este caso la madre) que confirma, plenamente al chico que su padre es negativo con él.

Resumiendo, son cinco los temas relacionales que están en la raíz del trastorno antisocial.

1) Apego evitativo sucesivamente reorganizado en una relación con los padres caracterizada por actitudes de exaltación, e hiperresponsabilización del hijo.

2) Rechazo paterno

3) Incapacidad para hacer respetar las reglas. Relaciones familiares igualitarias/simétricas entre los padres y el hijo.

4) Instrumentalidad y/o competitividad de las relaciones familiares en ausencia de dimensiones empáticas y de colaboración (no existe lealtad familiar).

5) Una vivencia de víctima por parte del paciente, alimentada por la presencia de uno o más "instigadores".

Mi deseo y mi previsión es que el futuro de la psicoterapia pueda construirse en la investigación sobre las raíces relacionales de los trastornos de la personalidad (o de los rasgos disfuncionales de la personalidad), la comprensión de los factores de descompensación de un trastorno de la personalidad, es decir, de los factores que producen una sintomatología psicopatológica clasificable en el primer eje del DSM IV.

Estudios de seguimiento

Voy ahora al segundo tema que quería tratar. Aprender de los errores es posible si estudiamos con atención los efectos de nuestras intervenciones pasadas.

Desde hace dos años aproximadamente, con un grupo de colaboradores de la Scuola de Psicoterapia della Famiglia di Milano, recientemente nominada con el nombre de su fundadora Mara Selvini Palazzoli, estamos trabajando en el seguimiento de todos los casos de psicosis tratados por la misma Selvini y sus diferentes equipos. Esta investigación sigue un trabajo análogo sobre la anorexia, iniciado con la colaboración de Mara Selvini en el año 1996 y publicado en el 1998 en el libro Muchachas anoréxicas y 
bulímicas (1). Con este breve artículo anticipamos los primeros datos del seguimiento de los pacientes tratados con el método paradojal en los años 70. (véanse tablas 1 y 2 )

\section{Nuestra reacción a estos datos}

No puedo ciertamente negar la primera reacción de tristeza y desánimo, mía y de todo el equipo de investigación frente a resultados tan desalentadores.

Algunas terapias entre las más creativas y brillantes, a veces también descritas en el libro Paradoja y Contraparadoja (10), habían concluido con progresos óptimos del paciente y grandes esperanzas. Hoy descubrimos que aquellos casos habían engañado a Mara Selvini y a sus colaboradores, porque, a lo largo de los años habían sido fuertes recaídas y sucesivas trágicas cronificaciones de las cuales nunca se había sabido nada.

Al mismo tiempo, estos datos confirmaban dudas que teníamos desde hacía mucho tiempo: en efecto el método paradójico se había abandonado desde hacía mas de 20 años , porque se había entendido que no se podía basar la terapia familiar en la provocación pura, en un contexto demasiado "agonístico" con las familias, donde la alianza terapéutica era demasiado contradictoria desde el punto de vista de la transparencia y la autenticidad.

\section{Importancia del diagnóstico individual}

Pero ahora estos datos nos confirman una esencial premisa teórica en la cual nunca ha dejado de creer Mara Selvini: la absoluta relevancia del diagnóstico individual del paciente. Selvini nunca ha desligado sus observaciones científicas de las definiciones diagnósticas de los pacientes de los que hablaba: anorexia, psicosis, etc. Esta premisa no ha sido siempre considerada históricamente en el mundo de la terapia familiar y de la psiquiatría antimanicomial de la cual nació la terapia familiar europea.

Un pionero como Haley (11), ya hace muchos años, afirmó que, para el terapeuta familiar, el diagnóstico individual del paciente no sólo es inútil sino que también es dañino. Muchos, desgraciadamente, le han seguido en este terreno.

También Mara Selvini tendía a ignorar el diagnóstico del paciente en el curso de los tratamientos individuales. Lo recuperaba al nivel de la reflexión científica como lo demuestran sus brillantes estudios sobre el mundo personal e interpersonal de la joven anoréxica, con una reflexión sobre los determinantes sociales todavía actuales. Predominaba en ella la idea de restituir al paciente el sentido de su absoluta libertad de elección (12). Ante el paciente Mara Selvini no creía en su "patología", no aceptaba sus límites, no toleraba que se pusiera como una víctima impotente. Esta es la filosofía de fondo de los protocolos de la terapia sistémica paradójica (véase tabla 3)

Sin embargo estos datos de investigación retrospectiva nos ponen de frente con impresionante evidencia la diversidad del impacto a largo plazo del mismo protocolo 
terapéutico, efectuado en el mismo año, en el mismo lugar, por el mismo equipo, sobre pacientes con diagnostico individual de anorexia respecto a pacientes con diagnóstico individual de psicosis. Las chicas anoréxicas han recibido de las intervenciones paradójicas un primer impulso eficaz que las ha encaminado hacia un curso de cambio que lleva a la recuperación en mas del $90 \%$ de los casos. Esta impresionante diferencia de resultados no es banal. En efecto muchos estudios en el campo de la denominada evidence based medecine o empirically supported treatment son criticados porque no tienen en cuenta la subjetividad del terapeuta. Esta confrontación muestra resultados diversos aunque con la misma subjetividad.

Conclusiones análogas pueden valer para la confrontación de cambios específicos y no específicos en psicoterapia. Estos datos muestran la gran importancia de factores específicos y por ello del impacto entre un cierto tipo de protocolo y una específica área diagnosticada en un determinado contexto relacional. (véase tabla 4)

Los chicos psicóticos han recibido un impulso que ha producido a veces progresos significativos en el mismo curso de la terapia, pero su éxito a largo plazo ha permanecido trágico, en línea con los datos de otras históricas investigaciones de seguimiento: solo $1 / 3$ de los esquizofrénicos llegan a una recuperación al menos social, mientras que cerca de $2 / 3$ de los pacientes graves no esquizofrénicos llegan a una recuperación al menos social (13)

\section{La investigación de evaluación de resultados es indispensable}

Estos datos proponen de nuevo el imperativo científico y ético de que los modelos terapéuticos no pueden ser usados prescindiendo del diagnóstico individual del paciente, siguiendo anárquicamente las preferencias personales del terapeuta o siguiendo simplemente las directivas propuestas por su modelo formativo demasiado arbitrariamente. Necesitamos datos lo más ciertos posibles que nos demuestren qué modelos y protocolos terapéuticos funcionan con qué tipo de pacientes, con qué tipo de familias, en qué contexto. Si la psicoterapia no acepta este presupuesto se arriesga seriamente a la desaparición o la marginación.

Las investigaciones retrospectivas como la nuestra o las del grupo de Andolfi (14) aunque son de gran interés, no son sin embargo suficientes.

\section{El London depresión intervention trial}

El movimiento de la terapia familiar debe agradecer fuertemente a Julian Leff y su equipo del Institute of Psychiatry en Londres, el haber ayudado a la psicoterapia y a la terapia sistémica a adquirir un nivel científico importante. Estoy hablando del London Depresión Intervention Trial surgido en el 1991 para comparar la eficacia de los fármacos antidepresivos con la terapia cognitiva individual y con la terapia sistémica de pareja.

"Los pacientes diagnosticados de depresión por un psiquiatra venían asignados con método casual a una de estas tres modalidades de tratamiento. La parte de la 
investigación concerniente a la terapia cognitiva se tuvo que interrumpir porque los abandonos eran demasiado abundantes (8 de 11 casos). Por eso la comparación final se pudo hacer entre los fármacos y la terapia sistémica de pareja: el descubrimiento más importante fue que la terapia sistémica daba resultados mucho mejores que los fármacos" (2 p.3.)

Debemos agradecer de todo corazón a los terapeutas Eia Asen y Elsa Jones (15) por haber participado con gran implicación en esta investigación, haber preparado un protocolo que describe con precisión qué es la terapia sistémica de pareja y haber demostrado de esta manera tan brillante su eficacia (añadiendo la condición de debilidad debida a la ausencia de un equipo terapéutico)

\section{El rol de las asociaciones nacionales y europeas de terapia familiar}

Creo que es tarea fundamental de las asociaciones trabajar con las entidades públicas de investigación nacionales e internacionales para que se organicen por toda Europa otros trial que comparen la eficacia de los protocolos sistémicos con otras intervenciones tradicionalmente utilizadas con la esquizofrenia, la anorexia, la drogodependencia etc. Nuestro desafío, como lo fue para Asen y Jones, es el de proyectar detalladamente protocolos de terapia que puedan ser llevados a la práctica. Las ideas están, basta con ver en el campo de la depresión el libro recientemente publicado por Linares y Campo (16).

Al mismo tiempo no creo que nuestras asociaciones puedan ser demasiado dependiente de otras entidades de investigación. Las asociaciones profesionales de psicoterapia y psiquiatría deben proporcionar instrumentos estándar para definir, diagnosticar y cuantificar por gravedad los problemas que nosotros, profesionales acreditados e inscritos a las asociaciones, afrontamos en nuestra actividad psicoterapéutica en los diversos contextos. Estos datos irían introducidos en un sistema informativo nacional y europeo. De este modo, podríamos saber en cada momento sobre qué población de usuarios estamos trabajando como profesionales y como evoluciona en el tiempo nuestra población clínica.

Sucesivamente se introducirían datos esenciales respecto al tipo de tratamiento puesto en práctica (descripción del protocolo terapéutico). A distancia de 2 o 3 años, siempre siguiendo las indicaciones estándar definidas, se procedería a las entrevistas de seguimiento relativas al estado del problema, a los índices de eficacia del tratamiento y al recuerdo que tienen los pacientes de la terapia.

Grupos de investigación independientes de las asociaciones deberían encargarse de revisar las muestras con un método de control casual con objeto de que los tratamientos efectivos correspondan al protocolo terapéutico descrito y que los resultados obtenidos no estén alterados

Los procedimientos de control son esenciales para garantizar la credibilidad de los datos, no solo para la comunidad científica, el público y las instituciones, sino también para nosotros mismos. Es evidente que solo los datos relativos al funcionamiento de centenas de casos pueden tener un interés en ausencia de grupos de comparación. 


\section{Una primera interpretación de nuestros estudios retrospectivos}

Para concluir vuelvo a los datos del seguimiento de las anoréxicas y los psicóticos de los cuales he partido. ¿Cómo explicamos la microscópica diferencia de resultados que hemos encontrado en nuestros estudios retrospectivos sobre el método paradójico de los años 70 ?

1) La primera respuesta es la mas obvia: los pacientes psicóticos tienen límites y dificultades personales mucho más graves que las chicas anoréxicas. No solo tienen menos recursos para el cambio sino también sus familias tienen menos recursos terapéuticos. El dato más lineal y elemental es la presencia de serios problemas psicológicos verificados en los padres y en los hermanos, cuya frecuencia es verificada en un porcentaje de $4 / 5$ veces superior en nuestras familias con chicos psicóticos respecto a nuestras familias de anoréxicas (17).

En las familias de las anoréxicas la negación de la patología aumenta en las pacientes la confianza en ellas mismas y atenúa las actitudes de control de los padres. Y esto las hace mejorar. Por el contrario, en las familias de los psicóticos la negación de la patología pone en riesgo de oscurecer el sufrimiento de estos chicos incitando así una mayor hostilidad e intolerancia de sus familiares. Esta dinámica resulta evidente tras la lectura de algunas terapias de los años 70 .

2) Una segunda respuesta, naturalmente relacionada con la primera, es relativa al tipo de protocolo de terapia breve (máximo 10 sesiones) provocadora y conjunta.

Las familias de los psicóticos requieren una toma de contacto más prolongada en el tiempo y no ligada solamente al formato familiar conjunto. Al releer hoy muchas terapias del equipo de Mara Selvini, quedamos desolados. Vemos por ejemplo, que con 10 sesiones se habían obtenido los primeros resultados, los familiares habían establecido una buena relación de confianza y alianza terapéutica, y pedían ayuda también como individuos. Justo en aquel momento, donde hoy pensaríamos que el verdadero proceso de cambio terapéutico estaría por comenzar, en obediencia al dictamen de la brevedad, provocación, fe absoluta en la capacidad autoterapéutica de los sistemas, estas familias eran despedidas para siempre, a pesar de su desconcertante protesta.

3) Además, bajo el nivel de la organización general de los dos tipos de familias, podríamos quizás generalizar diciendo que casi invariablemente las familias de las anoréxicas restrictivas se caracterizan por una fuerte intromisión recíproca de elevada intensidad emotiva, especialmente sobre el tema del control recíproco. Son raras, especialmente en el contexto de un centro de terapia familiar, las familias poco implicadas y desligadas. Al contrario, una parte de nuestra población de familias con un chico psicótico, aunque seguramente minoritaria, tiene estas características. Y son también muchas más las familias que han perdido la esperanza de recuperación. En los años 70 eran por consiguiente despedidas después de 1-2 sesiones. Se intentaba una intervención paradójica desesperada en el mismo acto de la despedida.

Las familias de los psicóticos requieren sin embargo un gran empeño e implicación del terapeuta para reconstruir los lazos familiares de empatía y colaboración. 
El mismo empeño es también importante pero no tan absolutamente esencial con las familias de las anoréxicas, porque estas chicas pueden reaccionar positivamente a un desafío que valore su capacidad.

4) Una última hipótesis de explicación tiene que ver con la experiencia de 20 años de terapia individual de las anoréxicas que llevaba Mara Selvini en el momento en que inició a experimentar el método paradójico. Sin embargo, su experiencia con la psicosis era enormemente inferior.

Releyendo casi 30 años después las terapias familiares de las anoréxicas aparecen mucho más específicas, originales y creativas que muchas de las terapias de los psicóticos. Podemos entonces pensar que el conocimiento profundo de la anorexia consintió a Selvini construir hipótesis relacionales e intervencionistas mucho más específicas. El método paradójico fue construido a medida para la paciente anoréxica y luego fue indebidamente alargado a las familias con pacientes psicóticos.

Mara Selvini, en su libro La Anorexia Mental (Self-Starvation) (6) describe con mucha eficacia cómo la personalidad de la anoréxica, incluso la de aquellas aparentemente más sumisas e inhibidas, se caracteriza por un elemento de fuerza y de oposición que ella llamaba l'artiglio stenico ("garra de fuerza" en español)". Este aspecto es el que impide a la paciente sucumbir a la depresión consecuente del sentimiento que en ella misma "hay un defecto". (Esta misma observación es compartida con V. Ugazio (18), que define como polaridad semántica fundamental en la familia de la anoréxica la que va entre el poder y la impotencia). Ahora la contraparadoja tiene su blanco y su punto de fuerza en esta garra de fuerza: ordenar a un paciente que prosiga en su comportamiento sintomático puede tener efecto solo si en el mismo paciente hay un fuerte componente de desafío y contraposición ("no cederé, no me obligaréis a comer"). Pero este aspecto muy raramente se presenta en el psicótico, que está normalmente hundido en el sentimiento de desesperación y fracaso, donde el componente de control del ambiente está ausente o estrictamente secundario.

\section{Conclusiones}

Esta última observación de nuevo me lleva al diagnóstico, y a la utilidad para la formación del profesional de la psicoterapia, de construirse una competencia especializada en el sector en el que decidirá o se encontrará trabajando.

La humanización de la psiquiatría es la batalla por la cual Mara Selvini Palazzoli y generaciones de psiquiatras y psicoterapeutas, han empleado su vida. Nos toca ahora a nosotros, con nuevas ideas y conocimientos, continuar la inevitablemente incompleta obra de nuestros pioneros. 


\section{TABLA 1}

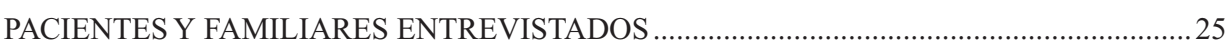

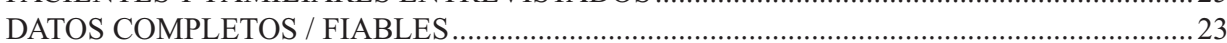

HOMBRES

17 MUJERES

.8

DIAGNOSTICO:

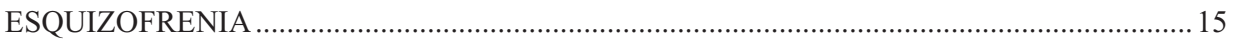

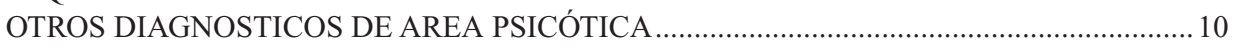

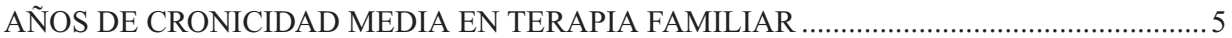

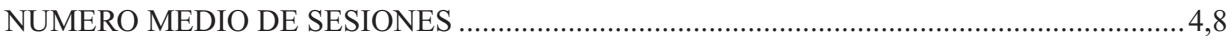

(del 1973 al 1979)

TABLA 2

BIENESTAR ACTUAL DE LOS PACIENTES:

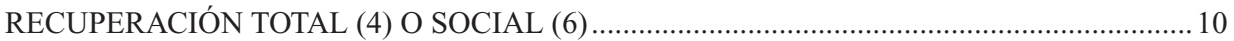

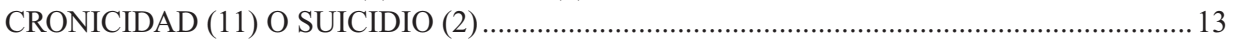

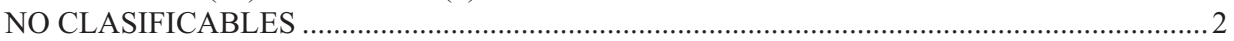

EFICACIA DE LA TERAPIA FAMILIAR:

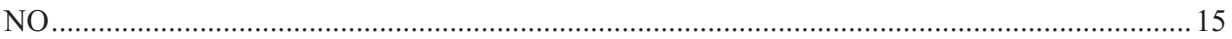

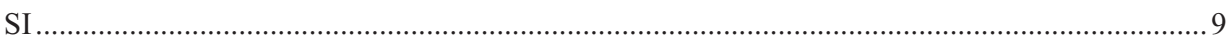

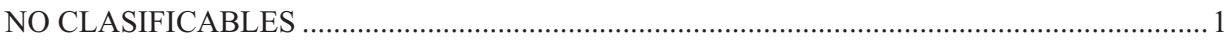

TABLA 3

SEGUIMIENTO ANOREXIA

(DEL LIBRO “MUCHACHAS ANOREXICAS Y BULÍMICAS”)

42 MUCHACHAS ( 31 DEL TIPO RESTRICTIVO) TRATADAS

CON EL MÉTODO PARADÓJICO EN LOS AÑOS 70

BIENESTAR ACTUAL:

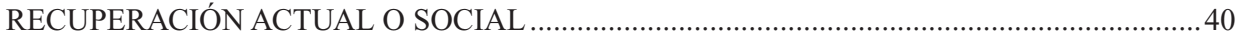

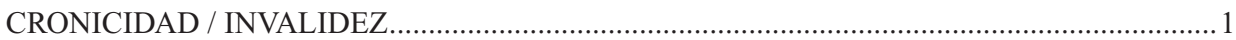

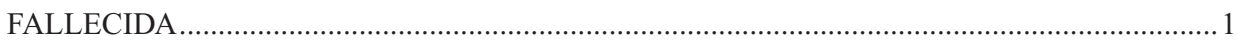

EFICACIA DE LA TERAPIA FAMILIAR

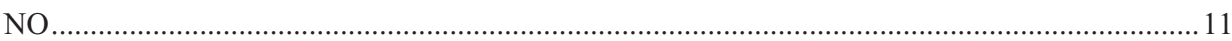

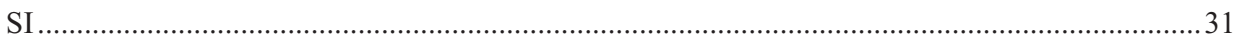




\section{TABLA 4}

CORRELACIÓN ENTRE EL BIENESTAR ACTUAL Y EL DIAGNOSTICO

- DE 15 ESQUIZOFRÉNICOS 10 SON CRÓNICOS Y 5 SOCIAL O TOTALMENTE RECUPERADOS

- DE LOS 8 PSICÓTICOS NO ESQUIZOFRÉNICOS 1 ES CRÓNICO, 2 SE HAN SUICIDADO, 5 SOCIAL O TOTALMENTE RECUPERADOS

- CORRELACIÓN ENTRE LA EFICACIA DE LA TERAPIA FAMILIAR Y EL DIAGNOSTICO:

- RESPECTO A LOS 15 ESQUIZOFRÉNICOS NO SE HA OBTENIDO EFICACIA EN 9 CASOS Y SE HA OBTENIDO EFICACIA POSITIVA EN 6 CASOS

- RESPECTO A LOS 9 PSICÓTICOS NO ESQUIZOFRÉNICOS NO SE HA OBTENIDO EFICACIA EN 6 CASOS Y SE HA OBTENIDO EFICACIA POSITIVA EN 3 CASOS

Agradecimientos:

En el trabajo interesante, pero fatigoso, de las entrevistas de seguimiento ha sido fundamental la colaboración de los colegas y colaboradores de la escuela de Psicoterapia de la familia de Milán "Mara Selvini Palazzoli": Simona Benzi, Gloria Bova, Stefania Capelli, Franca Do, Daniela Fabrizzi, Sara Ferraris, Elvira Fernández, Andrea Gazziero, Paola Morosini, Styliano Nicoalou, Emanuela Pasin, Alberto Penna, Antonio Pepoli.

\section{BIBLIOGRAFIA}

1.- Selvini Palazzoli, M., Cirillo, S., Selvini, M. Sorrentino, A.M. (1998) Muchachas anoréxicas y bulímicas, Paidos, Barcelona 1999.

2.- Selvini Palazzoli, M., Cirillo, S., Selvini, M., Sorrentino, A.M. (1988) Los juegos psicoticos en la familia, Paidos, Barcelona 1990.

3.- Lidz, T., Fleck, S., Cornelison, A., Schizophrenia and the family, International Universities Press, Nueva York, 1965.

4.- Haley, J. "La famiglia dello schizofrenico: un sistema modello", Journal of Nervous and Mental Disease, 1959, 129, págs. 357-374.

5.- Minuchin, S., Rosman, B.L., Baker, L. Famiglie psicosomatiche. L'anoressia mentale nel contesto familiare, Astrolabio, Roma 1980.

6.- Selvini Palazzoli, M. Self-Starvation, Chaucer Londres, (1974) tr. it. L'anoressia mentale, Feltrinelli, Milano (1981).

7.- Guidano, V., La complessità del Sé. Un approccio sistemico-processuale alla patologia e alla terapia cognitiva, Turin, Bollati Boringhieri, 1988.

8.- Miller, A. (1981) Il bambino inascoltato, Turin, Bollati Boringhieri, 1989. 
9.- Cirillo, S., Rangone, G., Selvini, M. (1997) "El subtipo regular de la familia del adolescente antisocial", REDES, 3, pp. 29-42.

10.- Selvini Palazzoli, M., Boscolo, L., Cecchin, G.F. Prata, G. Paradoja y contraparadoja, Paidos, Barcelona 1989.

11.- Haley, J. "A new phenomenon" in Zeig, J. (Eds.) The evolution of psychotherapy, BrunnerMazel, New York. 1987

12.- Selvini, M. "Mara Selvini Palazzoli: un tipico caso di resilienza" (in corso di stampa su Terapia Familiare). 2001

13.- McGlashan, T.H., Keats, C.J. Schizofrenia, Cortina, Milano: 1989

14.- Andolfi, M., Angelo, C., D’Atena, P. La terapia narrata dalle famiglie, Milano, Cortina, 1989

15.- Asen, E., Jones, E. Systemic couple therapy and depression, Londres, Karnac, 2000

16.- Linares, J.L., Campo, C. Tras la honorable fachada, Barcelona. Paidós. 2000

17.- Selvini, M., Benzi, S., Capelli, S., Ferraris, S., Gazziero, A., Morosini, P., Pasin, M., Penna, A., Pepoli, A. "Gli psicotici trattati da Mara Selvini Palazzoli e dalla sua équipe negli anni '90”, Ecologia della Mente, 2001. Giugno, pp. 47-53.

18.- Ugazio, V. Storie permesse e storie proibite, Torino. Bollati Boringhieri. 1998

(*) Co-fundador y responsable de la Escuela de Psicoterapia de Familia de Milán "Mara Selvini Palazzoli”.

Dirección para correspondencia:

Le Vittorio Veneto, 12

Milano - Italia

e-mail: matselvi@hotmail.com

Fecha de recepción: 12-04-02 